\title{
Characterization and Identification of The Key Residues Abound The Activity Site Tunnel of Trehalose Synthase from Pseudomonas Stutzeri Qlu3
}

\author{
Jing SU \\ Faculty of Light Industry, Province Key Laboratory of \\ Microbial Engineering, Qilu University of Technology \\ Jinan, China \\ e-mail: sj_12346@163.com
}

\section{Zhenzhen LI}

Faculty of Light Industry, Province Key Laboratory of Microbial Engineering, Qilu University of Technology Jinan, China e-mail:910484922@qq.com

\section{Lizhen HAO}

Faculty of Light Industry, Province Key Laboratory of Microbial Engineering, Qilu University of Technology Jinan, China e-mail: 934908340@qq.com

\author{
Yunxiao ZHANG \\ Faculty of Light Industry, Province Key Laboratory of \\ Microbial Engineering, Qilu University of Technology \\ Jinan, China \\ e-mail: 453740008@qq.com
}

\section{Piwu LI}

Faculty of Light Industry, Province Key Laboratory of Microbial Engineering, Qilu University of Technology Jinan, China e-mail: piwuli@126.com

\section{Haijie ZHENG}

Faculty of Light Industry, Province Key Laboratory of Microbial Engineering, Qilu University of Technology Jinan, China e-mail: 750737880@qq.com

\section{Ruiming WANG* \\ Faculty of Light Industry, Province Key Laboratory of Microbial Engineering, Qilu University of Technology Jinan, China e-mail: 550474939@qq.com}

Jing Su, Yunxiao Zhang and Zhenzhen Li contributed equally to this work

\begin{abstract}
The conversion of maltose into trehalose has important applications in the manufacture of food and other products. The enzyme trehalose synthase (TreS) catalyzes the interconversion of maltose and trehalose with glucose as a byproduct. In this study, treS was cloned from Pseudomonas stutzeri Qlu3 genomic DNA. We predicted the structural characteristics of recombinant TreS bound to its substrate by using homology modeling and flexible docking studies of the enzyme-substrate system. These analyses showed six amino acids (Phe115, Phe255, Arg292, Asp403, Asp294, and Glu338) that interact extensively with the substrate during catalysis. In addition, an enclosed active site tunnel was revealed that controls substrate movement during intramolecular isomerization. Disruption of the tunnel by removing two loops led to total loss of isomerization activity. The A309E mutant showed increased isomerase activity and decreased hydrolase activity. In contrast, the Q219R, T308E, and L341Q mutants showed decreased isomerase activity and increased hydrolase activity. These results suggest that the size of the tunnel can influence isomerase activity and hydrolase activity. Therefore, our results exhibited the TreS from Pseudomonas stutzeri Qlu3 have potential industrial use and the analysis in the structure has additional aid for the further molecular modification.
\end{abstract}

Keywords-characterization; homology modeling;
Pseudomonas stutzeri Qlu3; trehalose synthase

\section{INTRODUCTION}

Trehalose is a non-reducing disaccharide that contains two glucose moieties linked by an $\alpha, \alpha-1,1$-glycosidic linkage. It is present in a variety of organisms, including bacteria, archaea, yeast, fungi, insects, and numerous other invertebrates [1]. It plays important roles as a carbon energy reserve, a protective agent under stress conditions, and a structural component of the cell wall [2]. Trehalose has similar protective effects in vitro; therefore, it has broad applications in the manufacturing of food, cosmetics, and pharmaceuticals $[1,3]$.

To date, three metabolic pathways for the biosynthesis of trehalose in microorganisms have been reported. The first pathway is catalyzed by two enzymes, trehalose-6-phosphate synthase and trehalose-6-phosphate phosphatase [4]. The second pathway involves the rearrangement of internal glycosidic linkages between the molecules in glucose polymers, such as maltooligosaccharides [5] The third pathway also involves internal rearrangement, specifically the reversible interconversion of maltose and trehalose, which is catalyzed 
by trehalose synthase (TreS) [6]. Thus, TreS is considered as a convenient and practical biocatalyst for the industrial production of trehalose because of the one-step reaction and low cost of the maltose substrate. TreS has been purified from many other microorganisms and characterized [7-11]

Pseudomonas stutzeri is a metabolically versatile saprophytic soil bacterium that exhibits extensive biotransformation potential and efficiently produces a wide range of bulk and fine chemicals. TreS was isolated from Pseudomonas stutzeri CJ38 and exogenously expressed in E. coli BL21 (DE3) [10]. However, there is currently insufficient knowledge regarding the structural characteristics of this enzyme, including substrate specificity and transition state information.

In the present study, we sought to better understand the structure and substrate specificity of TreS. The P. stutzeri Qlu3 TreS protein is 689 amino acid residues in length with a molecular weight of $75 \mathrm{kDa}$. The conversion of maltose to trehalose by recombinant TreS had $\mathrm{pH}$ and temperature optima of 7.5 and $35^{\circ} \mathrm{C}$, respectively. At a maltose concentration of $20 \%$, the maximum conversion rate was greater than $70 \%$, with low byproduct production $(3.2 \%)$. To better understand the mechanism of catalysis, we characterized the structure of TreS through homology modeling and active site analysis with AutoDock. Then, we used site-directed mutagenesis to generate several mutants based on the AutoDock results and compared the activity of the mutant and wild-type enzymes.

\section{MATERIALS AND METHODS}

\section{A. Bacterial Strains, Chemicals, Media, and Culture Conditions}

P. stutzeri Qlu3 was maintained in our laboratory. E. coli BL21 (DE3) was cultured at $37^{\circ} \mathrm{C}$ in lysogeny broth (LB) and was used as the expression host. All chemicals were purchased from Sigma (St. Louis, MO, USA).

\section{B. Gene Cloning and Oligonucleotide Directed Mutagenesis Sequencing}

P. stutzeri Qlu3 genomic DNA was purified by using a bacterial genome DNA extracting kit (Takara), and the resultant DNA was used as the template for PCR amplification of the treS gene. The amplified treS gene was cloned into the BamHI and XhoI sites of pET15b (Novagen) in frame with an N-terminal $6 \times$ His-tag, and the resulting plasmid was transformed into $E$. coli BL21 (DE3) cells for TreS overexpression. Thirteen TreS mutants (F115A, H154A, Q219A, F255A, R292A, N340A, D403A, D294A, E338A, Q219R, T308E, A309E, and L314Q) were generated by using a two-step PCR strategy, and the sequences were confirmed by sequencing (Invitrogen). The primers used for PCR amplification and mutagenesis are shown in Supplementary Table II.

\section{Protein Expression and Analysis of TreS Activity}

E. coli BL21 (DE3) harboring the treS overexpression plasmid was grown in LB medium containing $100 \mu \mathrm{g} / \mathrm{mL}$ ampicillin until the $\mathrm{OD}_{600}$ reached 0.9 . TreS overexpression was induced by adding IPTG $(0.12 \mathrm{mM}$, final concentration $)$ and incubating overnight at $15^{\circ} \mathrm{C}$. Cells were subsequently harvested by centrifugation and lysed by ultrasonication. After centrifugation at $28,000 \times g$ for $45 \mathrm{~min}$, the supernatant was applied to a Ni-NTA column (GE Healthcare). The $6 \times$ His-tagged TreS was eluted with elution buffer $(25 \mathrm{mM}$ Tris- $\mathrm{HCl}, \mathrm{pH} 8.0,100 \mathrm{mM} \mathrm{NaCl}$, and 250 $\mathrm{mM}$ imidazole). The protein was further purified by anion exchange on a Source-Q column (GE Healthcare) and by size-exclusion chromatography on a Superdex 200 column (GE Healthcare). Purified TreS was analyzed by SDS-PAGE followed by Coomassie blue staining.

The activity of TreS was quantified by measuring the trehalose yield. The amount of sugar produced after the enzymatic reaction was measured by HPLC using $75 \%$ acetonitrile and $25 \%$ double distilled water as the mobile phase. The conversion rate was measured as the ratio of the trehalose product to the initial amount of maltose substrate. The amounts of trehalose, maltose, and glucose were determined using a trehalose reference standard (purity $>99.5 \%$; Sigma).

\section{Biochemical Properties of Recombinant TreS}

The effects of $\mathrm{pH}$ on TreS activity were determined by performing the reactions in $20 \mathrm{mM}$ citric- $\mathrm{NaH}_{2} \mathrm{PO}_{4}$, $\mathrm{Na}_{2} \mathrm{HPO}_{4}-\mathrm{NaH}_{2} \mathrm{PO}_{4}$, or $\mathrm{NH}_{3}-\mathrm{NH}_{4} \mathrm{Cl}$ buffer systems, with $\mathrm{pH}$ ranges of 5.0-6.0, 5.5-8.0, and 8.0-11.0, respectively. The effects of temperature on enzyme activity were determined by varying the reaction temperature from $20^{\circ} \mathrm{C}$ to $60^{\circ} \mathrm{C}$. The $\mathrm{pH}$ - and temperature-stability of TreS were determined by incubation at the indicated $\mathrm{pH}$ values and temperatures for $30 \mathrm{~min}$. TreS activity was also assayed in the presence of metal ions and other chemical reagents (at $1 \mathrm{mM}$ and $10 \mathrm{mM})$, to determine the effect of these substances on enzyme activity. The experiments were performed in a $1 \mathrm{~mL}$ reaction volume containing $20 \%$ maltose, $20 \mathrm{mM}$ $\mathrm{Na}_{2} \mathrm{HPO}_{4}-\mathrm{NaH}_{2} \mathrm{PO}_{4} \quad(\mathrm{pH}=7.2)$, and $1 \mu \mathrm{M}$ purified recombinant TreS at $37^{\circ} \mathrm{C}$. Enzyme activity was assessed at $5 \mathrm{~min}, 20 \mathrm{~min}, 40 \mathrm{~min}, 1 \mathrm{~h}, 2 \mathrm{~h}$, and $3 \mathrm{~h}$.

\section{E. Homology Modeling and Flexible Docking of Substrate to TreS}

Homology modeling of TreS was performed by using I-TASSER, an online platform for protein structure and function prediction [12]. Molecular graphics were generated with PyMol (http://www.pymol.org/). AutoDock 4.2 [13]. was used to prediction of substrate docking with TreS. A grid box with sufficient margins $(40 \times 36 \times 36 \AA)$ was placed to restrain the substrate molecule, which covered the potential active site region of TreS. This potential active region was suggested by comparison to the structure of TreS from Deinococcus radiodurans, [14] a homologue of $P$. stutzeri TreS.

\section{RESULTS AND DISCUSSION}

\section{A. Cloning of The TreS Gene and pET-15b-treS Vector Construction}

As shown in Fig. 1A, the treS gene was amplified from 
P. stutzeri Qlu3 genomic DNA. A band of the appropriate size for treS was observed by agarose gel electrophoresis of the PCR sample. The treS PCR-amplified fragment was cloned into the pET-15b expression vector to generate pET-15b-treS.

\section{B. Evaluation of The Biochemical Properties of TreS}

TreS (as a $6 \times$ His-tagged fusion protein) was purified by using a Ni-NTA, Source-Q, and Superdex 200 columns. Purified TreS was analyzed by SDS-PAGE after Superdex 200 purification (fractions B11 to C7). As shown in Fig. 1B, a distinct band with a molecular mass of approximately 75 $\mathrm{kDa}$ was observed.

The temperature- and $\mathrm{pH}$-dependency of TreS activity on the conversion of maltose to trehalose were determined. The enzyme showed maximum activity at $35^{\circ} \mathrm{C}$ (Fig. 2A), and the enzyme retained $70 \%$ of its initial activity after heat treatment at $50^{\circ} \mathrm{C}$ for $30 \mathrm{~min}$ (Fig. 2B). The $\mathrm{pH}$ dependency was analyzed in $20 \mathrm{mM}$ citric- $\mathrm{NaH}_{2} \mathrm{PO}_{4}$, $\mathrm{Na}_{2} \mathrm{HPO}_{4}-\mathrm{NaH}_{2} \mathrm{PO}_{4}$, and $\mathrm{NH}_{3}-\mathrm{NH}_{4} \mathrm{Cl}$ buffer systems at $\mathrm{pH}$ ranges of 4.0-6.0, 5.5-8.0, and 8.0-11.0, respectively. TreS showed maximal activity between $\mathrm{pH} 7.5-8.5$ (Fig. 2C) and was stable in a pH range of 6.0-10.0 (Fig. 2D).

The effects of metal ions and reagents were analyzed at $1 \mathrm{mM}$ and $10 \mathrm{mM}$ concentrations of a variety of substances (Table I). When treated with $1 \mathrm{mM} \mathrm{Zn}{ }^{2+}, \mathrm{Cu}^{2+}$, or $\mathrm{Ni}^{2+}$, TreS activity was clearly inhibited. All other metals and reagents showed no obvious effects at this concentration. At $10 \mathrm{mM}$, inhibitory effects were observed for $\mathrm{Zn}^{2+}, \mathrm{Cu}^{2+}, \mathrm{Fe}^{2+}, \mathrm{Ca}^{2+}$, $\mathrm{Co}^{2+}, \mathrm{Mn}^{2+}, \mathrm{Ni}^{2+}$, and SDS. $\mathrm{Mg}^{2+}$ and $\mathrm{K}^{+}$showed no inhibitory effect.

As shown in Fig. 2E, approximately $70 \%$ of the maltose substrate was converted to trehalose after a 1-hr reaction. The reaction time was obviously shorter than previously reported [10]. The glucose byproduct yield was low, at $3.2 \%$

TABle I. EFFECTS OF METAL IONS AND REAGENTS ON THE ACTIVITY OF TRES

\begin{tabular}{cccccc}
\hline Reagent & \multicolumn{2}{c}{$\begin{array}{c}\text { Relative activity } \\
(\%)\end{array}$} & Reagent & \multicolumn{2}{c}{$\begin{array}{c}\text { Relative } \\
\text { activity }(\%)\end{array}$} \\
\cline { 2 - 3 } \cline { 5 - 6 } & $1 \mathrm{mM}$ & $10 \mathrm{mM}$ & & $1 \mathrm{mM}$ & $10 \mathrm{mM}$ \\
\hline none & $100 \pm 2$. & $100 \pm 3$. & $\mathrm{MgCl}_{2}$ & $101 \pm 4$. & $99 \pm 2.3$ \\
& 1 & 2 & & 5 & \\
$\mathrm{ZnSO}_{4}$ & $88 \pm 3.2$ & $12 \pm 2.3$ & $\mathrm{KCl}$ & $99 \pm 1.9$ & $99 \pm 3.3$ \\
$\mathrm{CuSO}_{4}$ & $89 \pm 1.6$ & $43 \pm 3.1$ & $\mathrm{CaCl}_{2}$ & $94 \pm 2.1$ & $66 \pm 4.1$ \\
$\mathrm{FeSO}_{4}$ & $94 \pm 2.7$ & $15 \pm 2.9$ & $\mathrm{CoCl}_{2}$ & $98 \pm 2.7$ & $64 \pm 2.1$ \\
EDTA & $99 \pm 0.9$ & $98 \pm 1.6$ & $\mathrm{NiCl}_{2}$ & $90 \pm 1.5$ & $87 \pm 1.9$ \\
SDS & $97 \pm 1.6$ & $34 \pm 2.6$ & $\mathrm{MnCl}_{2}$ & $99 \pm 2.2$ & $63 \pm 2.4$ \\
\hline
\end{tabular}

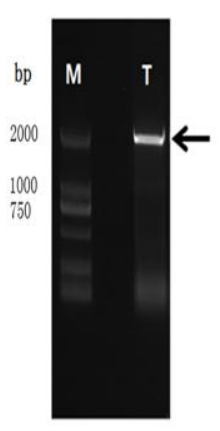

A

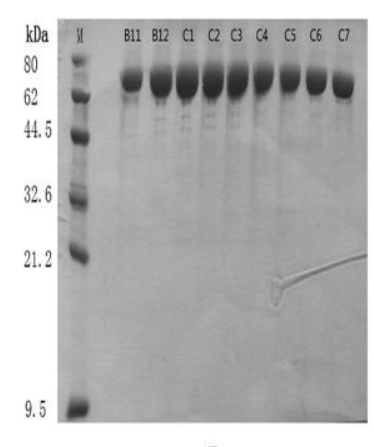

B
Figure 1. A Agarose electrophotresis result of TreS gene cloned from P.stutzeri qlu genomic DNA. The arrow shows the object band. B SDS-PAGE analysis of purified TreS by Superdex-200. B11 to C7 indicate the different tubules.

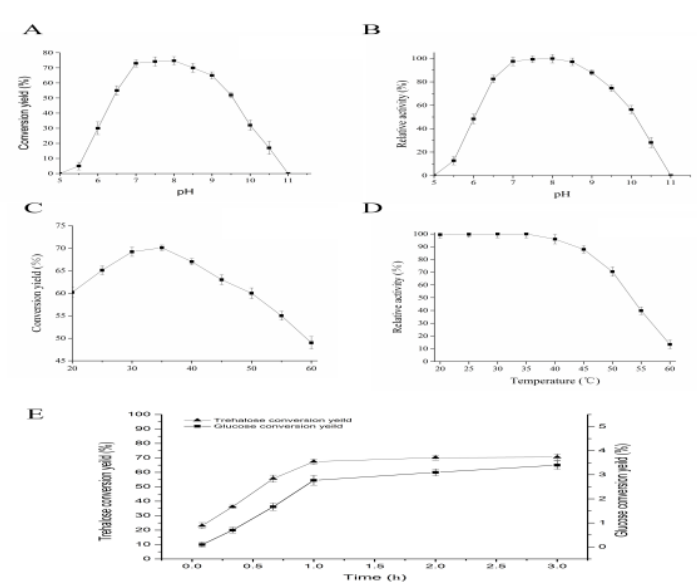

Figure 2. A show that the optimal temperature of the TreS activity is $35^{\circ} \mathrm{C}$. B show TreS retained $70 \%$ of its initial activity after heat treatment at $50^{\circ} \mathrm{C}$ for $30 \mathrm{~min}$. $\mathrm{C}$ show that the optimal $\mathrm{pH}$ of the TreS activity is 7.5-8.5. D show TreS retained stability at $\mathrm{pH}$ range of $6.0-10.0$ after $30 \mathrm{~min}$ of incubation. E Conversion yield of maltose to trehalose by TreS.

\section{Structure Prediction of The TreS Active Site and Analysis of Enzyme Activity}

The three-dimensional coordinates of the models were created by I-TASSER. The models of TreS from P. stutzeri Qlu3 showed a typical GH13 domain organization. The protein consisted of three domains: A, B, and C. To investigate the structure of the active site, we performed flexible docking of TreS and maltose by using AutoDock. The docked structure model revealed that the active site was located at the bottom of the A domain (Fig. 3A). Nine residues (F115, H154, Q219, F255, R292, N340, D403, D294, and E338) appear to be involved in substrate binding (Fig. 3B). To confirm the homologous modeling results, thirteen single amino acid mutants (F115, H154, Q219, F255, R292, N340, D403, D294, E338, Q219R, T308E, A309E, and L314Q) were generated by using a two-step PCR strategy, and the activities of the mutants were measured and compared to that of wild-type enzyme (Table IIA). The results showed that the F115A, F255A, R292A, 
D403A, D294A, and E338A mutants were inactive. In contrast, the activity of H154A was decreased by approximately $36.2 \%$ compared to that of wild type. Asp294 and Glu338 are highly conserved across the GH13 family and play an essential role in catalysis [15-16]. The loss of TreS activity due to mutation of Phe115, Phe255, Arg292, and Asp403 indicates that these amino acids also play important roles in substrate binding. Gln219 and Asn340 do not contact the substrate directly; therefore, as expected, mutations in these two residues only modestly affected enzymatic activity compared to the significant effects of the other seven mutations. These data are consistent with the results of the AutoDock calculations.

TABLE II. RELATIVES ENZYME ACTIVITIES OF WILD TyPE TRES AND Mutants. The ACTIVITY OF The WT (WILD TyPe) WAS SET To $100 \%$.

TABLE IIA

\begin{tabular}{ccc}
\hline Mutant(\%) & $\begin{array}{c}\text { Relative isomerase } \\
\text { activity }(100 \%)\end{array}$ & $\begin{array}{c}\text { Relative hydrolose activity } \\
(100 \%)\end{array}$ \\
\hline Wild type & $100.0 \pm 0.014$ & $100 \pm 0.102$ \\
D294A & $0.2 \pm 0.023$ & $0.12 \pm 0.015$ \\
F115A & $0.05 \pm 0.045$ & $0.08 \pm 0.032$ \\
F255A & $0.03 \pm 0.012$ & $0.01 \pm 0.005$ \\
R292A & $0.01 \pm 0.018$ & $0.09 \pm 0.012$ \\
D403A & $0.06 \pm 0.034$ & $0.12 \pm 0.035$ \\
H154A & $36.2 \pm 0.571$ & $41.8 \pm 1.031$ \\
N340A & $68.1 \pm 0.622$ & $56.8 \pm 0.976$ \\
Q219A & $95.6 \pm 1.981$ & $99.2 \pm 1.428$
\end{tabular}
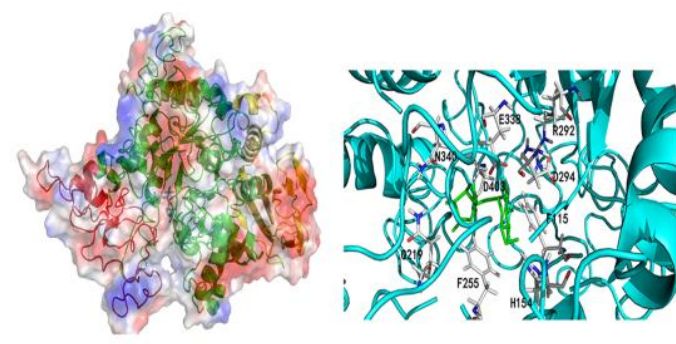

A
Figure 3. Structure prediction and the active site analysis A Substrate binding site predicted with autodock result. TreS is shown in cartoon model. The three domains of TreS (A, B, and C) are shown in green, red, and yellow respectively. The substrate is show in stick and sited in the narrow pocket of domain A. B The amino acids around the substrate predicted by Autodock. TreS is shown as cyan cartoon model. The substrate is shown as green stick. There are nine amino acids that interacted with the substrate: F115, H154, Q219, F255, R292, N340, D403, D294 and E338.

\section{Superpositioning with Homologs and Analysis of The} Activity Site Entrance

Examination of the structures of the homologous proteins MsTS [17] and DrTS [14] suggested that closure of the active site is the rate-limiting step in TreS-catalyzed trehalose conversion. The tight conformation of the structure limits substrate movement to promote intramolecular isomerization and minimize hydrolysis. In
DrTS, subdomain B (Asn105-Asn184) and S7 (Leu315-Asp361) seal the active site entrance [14]. When we superpositioned DrTS with TreS from P. stutzeri Qlu3, it revealed a similar arrangement, subdomain B (Ala153-Asp219) and S7 (Leu399-Ala472) in P. stutzeri TreS (Fig. 4A). In order to confirm the superposition results, we removed loop1 (Thr231-Asp243) in subdomain B and loop2 (Ala398-Val410) in S7. Removal of these loops resulted in a total loss of isomerase activity (Table IIB). Therefore, subdomains B and S7 play important roles in sealing the active site of TreS. Through superposition with DrTS, we determined the amino acids in subdomains B and S7 that interact, including Gln219, Thr308, Ala309, Asn340, Leu341, and Glu408. In order to improve the salt bridge interactions, we introduced four mutations, Q219R, T308E, A309E, and L341Q. The A309E mutant showed $\sim 104 \%$ of wild-type isomerase activity and $\sim 30.9 \%$ of wild-type hydrolase activity. In contrast, mutants Q219R, T308E, and L341Q showed decreased isomerase activity and increased hydrolase activity. These results suggest that the pore for water entry may be tighter in the A309E mutant.

Table II. RELATIVES ENZYME ACTIVITIES OF WILD TYPE TRES AND MUTANTS. THE ACTIVITY OF THE WT (WILD TYPE) WAS SET TO $100 \%$.

Table IIB.

\begin{tabular}{lcc}
\hline Mutant $(\%)$ & $\begin{array}{c}\text { Relative isomerase } \\
\text { activity }(100 \%)\end{array}$ & $\begin{array}{c}\text { Relative hydrolose activity } \\
(100 \%)\end{array}$ \\
\hline Wild type & $100.0 \pm 0.014$ & $100 \pm 0.102$ \\
Q219R & $98.3 \pm 1.357$ & $125.9 \pm 1.293$ \\
T308E & $97.5 \pm 0.956$ & $111.2 \pm 1.019$ \\
A309E & $104.5 \pm 0.939$ & $30.6 \pm 0.826$ \\
L341Q & $86.8 \pm 1.369$ & $328.2 \pm 0.751$ \\
\hline
\end{tabular}

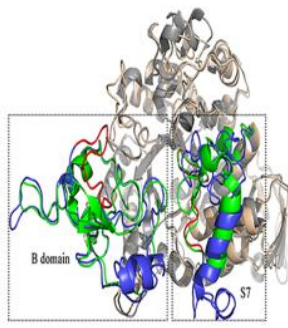

A

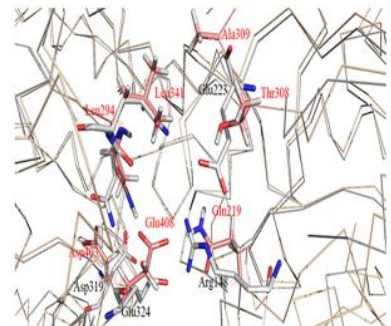

B
Figure 4. Stereoview of the structure superposition of TreS from P. stutzeri and DrTs (PDB entry 4tvu).(A) The structure were showed by cartoon model. The red loops represent removed in B domain and $\mathrm{S} 7$ from TreS from P.stutzeri. B domain and S7 of TreS from P.stutzeri colored in blue and other domains are in wheat color. B domain and S7 from DrTs colored in blue and other domains are in gray color. (B) Interaction networks between TreS from P. stutzeri (wheat) and DrTs (gray).

\section{CONCLUSION}

We showed here that the conversion rate of maltose to trehalose by P. stutzeri Qlu3 TreS was greater than 70\%, with low byproduct formation $(3.2 \%)$ after a $1-\mathrm{hr}$ reaction, making it a good candidate for large-scale production of trehalose. Sequence-based alignment showed that seven residues, D294, E338, F115, H154, F255, R292, and D403, 
are highly conserved across the GH13 family (Fig. 5). TreS has a similar catalytic mechanism to that of other GH13 family members. Asp294 plays an essential role as the nucleophile that attacks the anomeric center of the non-reducing sugar (maltose) in an acid-catalyzed process. Glu338 functions as an acid/base catalyst that attacks the glycosidic bond to form trehalose and regenerate the free enzyme. Another five amino acids, F115, H154, F255, R292, and D403, constitute the active site of TreS, which accommodates the substrate. Subdomains B and S7 play important roles in sealing the active site. The interaction between amino acids in subdomains B and S7 control the size of the pore for water entry. Our present study on TreS offers information that should aid in the modification of this enzyme for potential industrial use.

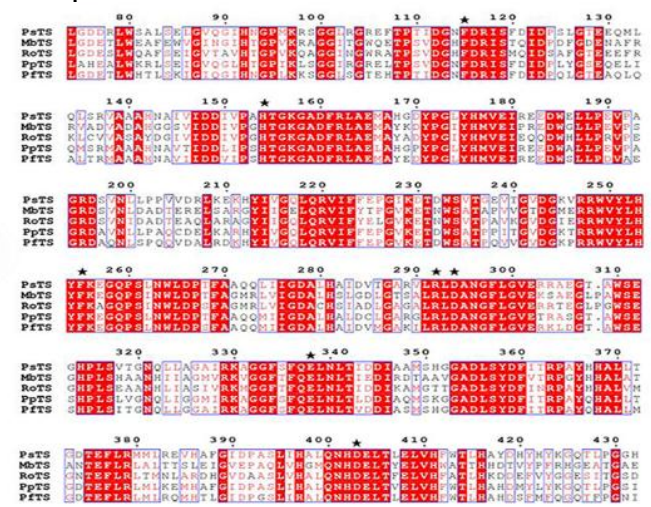

Figure 5. Multiple Sequence alignment of TreS. PsTS, Pseudomonas stutzeri Qlu3(study in the paper); MbTS, Microbacterium testaceum TreS (ref|WP_043363305.1); RoTS, Rhodococcus opacus TreS (gb|AII06508.1); PpTS, Pseudomonas putida TreS(ref|NP_745062.1); PfTS, Pseudomonas fluorescens TreS(ref|WP_034135059.1). The amino acids (D294, E338, F115, H154, F255, R292, and D403) are highly reserved and labeled with black asterisks.

\section{ACKNOWLEDGMENTS}

This work was supported by a grant from the National Natural Science Foundation of China (31401626) and Shandong Province Natural Science (ZR2014CQ039).

\section{REFERENCES}

[1] Elbein AD, Pan YT, Pastuszak I, Carroll D. "New insights on trehalose: a multifunctional molecule." Glycobiology. 2003; 13:17R-27R

[2] Carvalho AL, Cardoso FS, Bohn A, "Neves AR, Santos H. Engineering trehalose synthesis in Lactococcus lactis for improved stress tolerance." Applied and environmental microbiology .2011; 77:4189-99.

[3] Schiraldi C, Di Lernia I, De Rosa M. "Trehalose production: exploiting novel approaches." Trends in biotechnology.2002; 20:
420-5.

[4] Edavana VK, Pastuszak I, Carroll JD, Thampi P, Abraham EC, Elbein $\mathrm{AD}$. "Cloning and expression of the trehalose-phosphate phosphatase of Mycobacterium tuberculosis: comparison to the enzyme from Mycobacterium smegmatis." Archives of biochemistry and biophysics, 2004; 426:250-7.

[5] Nakada T, Ikegami S, Chaen H, Kubota M, Fukuda S, Sugimoto T, et al. "Purification and characterization of thermostable maltooligosyl trehalose synthase from the thermoacidophilic archaebacterium Sulfolobus acidocaldarius." Biosci. biotechnol. biochem. 1996; 60: 263-6.

[6] Nishimoto T, Nakano M, Nakada T, Chaen H, Fukuda S, Sugimoto T, et al. "Purification and properties of a novel enzyme, trehalose synthase, from Pimelobacter sp. R48. Biosci." biotechnol. biochem. 1996; 60: 640-4.

[7] Nishimoto T, Nakano, M., Ikegami, S., Chaen, H., Fukuda, S. Sugimoto, T., Kurimoto, M., and Tsujisaka, Y. "Existence of a novel enzyme converting maltose into trehalose." Biosci. Biotechnol. Biochem. 1995; 59: 2189-90.

[8] Chen YS, Lee GC, Shaw JF. "Gene cloning, expression, and biochemical characterization of a recombinant trehalose synthase from Picrophilus torridus in Escherichia coli." Journal of agricultural and food chemistry,. 2006; 54: 7098-104.

[9] Kim TK, Jang JH, Cho HY, Lee HS, Kim YW. "Gene cloning and characterization of a trehalose synthase from Corynebacterium glutamicum ATCC13032.” Food Sci Biotechnol. 2010; 19: 565-9.

[10] Lee JH, Lee KH, Kim CG, Lee SY, Kim GJ, Park YH, et al. "Cloning and expression of a trehalose synthase from Pseudomonas stutzeri CJ38 in Escherichia coli for the production of trehalose." Applied microbiology and biotechnology, 2005; 68: 213-9.

[11] Xiuli W, Hongbiao D, Ming Y, Yu Q. "Gene cloning, expression, and characterization of a novel trehalose synthase from Arthrobacter aurescens." Applied microbiology and biotechnology, 2009; 83: 477-82.

[12] Roy A, Kucukural A, Zhang Y. "I-TASSER: a unified platform for automated protein structure and function prediction." Nat Protoc. 2010; 5: 725-38.

[13] Morris GM, Goodsell DS, Halliday RS, Huey R, Hart WE, Belew $\mathrm{RK}$, et al. "Automated docking using a Lamarckian genetic algorithm and an empirical binding free energy function." J. Comput. Chem. 1998; 19: 1639-62.

[14] Wang YL, Chow SY, Lin YT, Hsieh YC, Lee GC, "Liaw SH. Structures of trehalose synthase from Deinococcus radiodurans reveal that a closed conformation is involved in catalysis of the intramolecular isomerization." Acta. Crystallogr D. 2014; 70: 3144-54.

[15] Su J, Wang TF, Ma CL, Li PW, Li ZK, Wang RM. "Homology modeling and function of trehalose synthase from Pseudomonas putida P06.” Biotechnol. Lett. 36 (2014) 1009-1013.

[16] Zhang R, Pan YT, He S, Lam M, Brayer GD, Elbein AD, Withers SG "Mechanistic analysis of trehalose synthase from Mycobacterium smegmatis." J. Biol. Chem. 286 (2011) 35601-35609.

[17] Caner S, Nguyen N, Aguda A, Zhang R, Pan YT, Withers SG, Brayer GD. "The structure of the Mycobacterium smegmatis trehalose synthase reveals an unusual active site configuration and acarbose-binding mode (dagger)." Glycobiology. 23 (2013) 1075-1083 
TABLE S1. THE PRIMERS USED IN THE PAPER

\begin{tabular}{|c|c|c|}
\hline Primer Name & Forward primer (5'-3') & Reverse primer (5'-3') \\
\hline TreS-wild & $\begin{array}{l}\text { ATCGGATCCATGAGCAHNCCAGACAAHAN } \\
\text { CTATATC }\end{array}$ & TCACTCGAGTTARANCACCGGHGR \\
\hline TreS D294A & $\begin{array}{c}\text { CGGGTGCTGCGCCTGGCAGCCAACGGCTTC } \\
\text { CTC }\end{array}$ & $\begin{array}{l}\text { GAGGAAGCCGTTGGCTGCCAGGCGCAGCAC } \\
\text { CCG }\end{array}$ \\
\hline TreS E338A & $\begin{array}{l}\text { GGCTTCAGCTTCCAGGCACTGAACCTGACC } \\
\text { ATC }\end{array}$ & $\begin{array}{l}\text { GATGGTCAGGTTCAGTGCCTGGAAGCTGAA } \\
\text { GCC }\end{array}$ \\
\hline TreS D403A & $\begin{array}{l}\text { GCCCTGCAGAACCATGCAGAGCTGACCCTG } \\
\text { GAG }\end{array}$ & $\begin{array}{l}\text { CTCCAGGGTCAGCTCTGCATGGTTCTGCAGG } \\
\text { GC }\end{array}$ \\
\hline TreS F115A & $\begin{array}{l}\text { ACCATCGACGGCAACGCAGACCGCATCAGC } \\
\text { TTC }\end{array}$ & $\begin{array}{c}\text { GAAGCTGATGCGGTCTGCGTTGCCGTCGATG } \\
\text { GT }\end{array}$ \\
\hline TreS F255A & $\begin{array}{l}\text { GTCTATCTGCACTACGCAAAGGAGGGCCAG } \\
\text { CCG }\end{array}$ & $\begin{array}{c}\text { CGGCTGGCCCTCCTTTGCGTAGTGCAGATAG } \\
\text { AC }\end{array}$ \\
\hline TreS R292A & $\begin{array}{c}\text { GGCGCCCGGGTGCTGGCACTGGACGCCAAC } \\
\text { GGC }\end{array}$ & $\begin{array}{l}\text { GCCGTTGGCGTCCAGTGCCAGCACCCGGGC } \\
\text { GCC }\end{array}$ \\
\hline TreS N340A & $\begin{array}{c}\text { AGCTTCCAGGAGCTGGCACTGACCATCGAT } \\
\text { GAC }\end{array}$ & $\begin{array}{c}\text { GTCATCGATGGTCAGTGCCAGCTCCTGGAAG } \\
\text { CT }\end{array}$ \\
\hline TreS Q219A & $\begin{array}{c}\text { ATCGTCGGCCAGCTGGCACGGGTGATCTTCT } \\
\text { TC }\end{array}$ & $\begin{array}{c}\text { GAAGAAGATCACCCGTGCCAGCTGGCCGAC } \\
\text { GAT }\end{array}$ \\
\hline TreS H154A & $\begin{array}{c}\text { CGACATCGTGCCGGCGCACACCGGCAAGGG } \\
\text { TGC }\end{array}$ & $\begin{array}{c}\text { GCACCCTTGCCGGTGTGCGCCGGCACGATGT } \\
\text { CG }\end{array}$ \\
\hline TreS Q219R & CATCGTCGGCCAGCTGAGACGGGTG & GAAGAAGATCACCCGTCTCAGCTG \\
\hline TreS T308E & CGGCGTGCCGAGGGCGAAGCCTGG & GCCCTCCGACCAGGCTTCGCCCTC \\
\hline TreS A309E & CGTGCCGAGGGCACCGAATGGTCG & GTGGCCCTCCGACCATTCGGTGCC \\
\hline TreS L341Q & TTCCAGGAGCTGAACCAAACCATC & GATGTCATCGATGGTTTGGTTCAG \\
\hline
\end{tabular}

